Supporting Information for:

\title{
Enzymatically Controlled Vacancies in Nanoparticle Crystals
}

Stacey N. Barnaby, ${ }^{1,2}$ Michael B. Ross, ${ }^{1,2}$ Ryan V. Thaner, ${ }^{1,2}$ Byeongdu Lee, ${ }^{3 *}$ George C. Schatz, ${ }^{1,2^{*}}$ and Chad A. Mirkin ${ }^{1,2^{*}}$

${ }^{1}$ Department of Chemistry, Northwestern University, 2145 Sheridan Road, Evanston, Illinois 60208, United States; ${ }^{2}$ International Institute for Nanotechnology, Northwestern University, 2145 Sheridan Road, Evanston, Illinois 60208, United States; ${ }^{3} \mathrm{X}$-ray Science Division, Argonne National Laboratory, 9700 South Cass Avenue, Argonne, IL 60439

*To whom correspondence should be addressed:

E-mail: chadnano@northwestern.edu

Phone: 847-467-7302

Email: g-schatz@northwestern.edu

Phone: 847-491-5657

Email: blee@aps.anl.gov

Phone: 630-252-0395

\section{SI Discussions}

Melting Analysis of the Crystals after Enzymatic Removal of Particle B'

To identify whether any $\mathrm{B}^{\prime}$ particles were still present in the crystal after addition of ribonuclease A (RNase A), melting analyses were conducted on the pellets in Figure 4b. A melting transition was observed at $\approx 52{ }^{\circ} \mathrm{C}$, which corresponds to the DNA/DNA bonding elements. We note the absence of the melting transition at $54{ }^{\circ} \mathrm{C}$ (observed in Figure $2 \mathrm{a}$ and $\mathrm{S} 1 \mathrm{c}, \mathrm{d}$ ), thus providing evidence that the RNA bonding elements are no longer present in the crystals. However, an additional melting transition is observed at 45 ${ }^{\circ} \mathrm{C}$ (Figure S2a). Previous work has identified that less cooperativity results in lower, broader melting temperatures; ${ }^{1}$ we hypothesize that an analogous effect could occur due to having under-coordinated particles with DNA bonding elements. This less cooperative melting behavior reflects that a given particle has fewer oligonucleotide linkages, which is likely due to some combination of having fewer nearest neighbors or fewer connections between particles. ${ }^{1}$ To probe this effect, we modified the two-state melting model by decreasing the extent of cooperativity (defined as the number of linker strands (100) divided by the number of nearest neighbors (8)) in 0.5 increments from 12.5 to 9 (Figure S2b). In all cases, the slope remained the same but a uniform decrease in melting temperature was observed, which likely suggests that there may be DNA/DNA melting transitions in environments that are less cooperative than before the RNA bonding elements were hydrolyzed. Despite the simplicity of the model, the theoretically generated curves for a cooperativity value of 12.5 agreed well with the experimentally measured melting curves. Simply decreasing the cooperativity to 11 drops the melting 
temperature to what is observed experimentally $\left(45^{\circ} \mathrm{C}\right)$ without changing the slope of the melting curve. Therefore, the theoretical melting curves generated using the two-state melting model agree well with the experimental observation of decreased melting temperatures in superlattices with increasing number of vacancies.

\section{Concentration of Nanoparticles Enzymatically Hydrolyzed from Crystals}

UV-visible spectroscopy was used to quantify the concentration of nanoparticles in the supernatants of Figure $4 \mathrm{~b}$ (Figure S3a; solid bars) in comparison to the amount of particle $\mathrm{B}^{\prime}$ added during synthesis of the superlattices (dashed bars). At $<40 \%$ particle B', there is good agreement between the amount of particle $\mathrm{B}^{\prime}$ added and the concentration of gold nanoparticles (AuNPs) measured in the supernatant. At $>80 \%$ particle $\mathrm{B}^{\prime}$, the concentration of AuNPs in the supernatant was greater than the number of $\mathrm{B}^{\prime}$ particles added. This correlates with the fact that the nanoparticles are no longer able to remain aggregated due to hydrolysis of a critical number of RNA bonding elements, so particles A or B must also be contributing to the optical signal. Indeed, this measurement is only one of the AuNP concentration in the supernatant; it does not take into account whether the AuNPs were particles A, B, or B'.

\section{Comparison of Static and In Situ Interaction of Nanoparticle Superlattices with RNase A}

The "static" SAXS experiments only provide a snapshot of the interaction of nanoparticle superlattices with RNase A after a set incubation time. In order to observe structural changes in real time, we used in situ flow cell SAXS, where we observed a gradual broadening of the spectrum and shift to larger interparticle distances over time (Figure S4). We note differences in interparticle distance observed for the in situ flow cell experiment (shift to larger interparticle distances; Figure S5) and the "static" experiment of analyzing the enzyme interactions after 12 hours (little to no change in interparticle distance; Figure 5a). We hypothesized that because the flow cell experiment enabled removal of particles from the lattice in real time, the lattice would not have time to fill the vacancies left behind by the enzymatically hydrolyzed nanoparticles. ${ }^{2}$ In addition, the in situ experiments were run over the course of 30 minutes, versus 12 hours for the static experiment. Therefore, in the static experiment, the superlattice has time to re-arrange to accommodate the strain in the system occurring as vacancies are generated. 


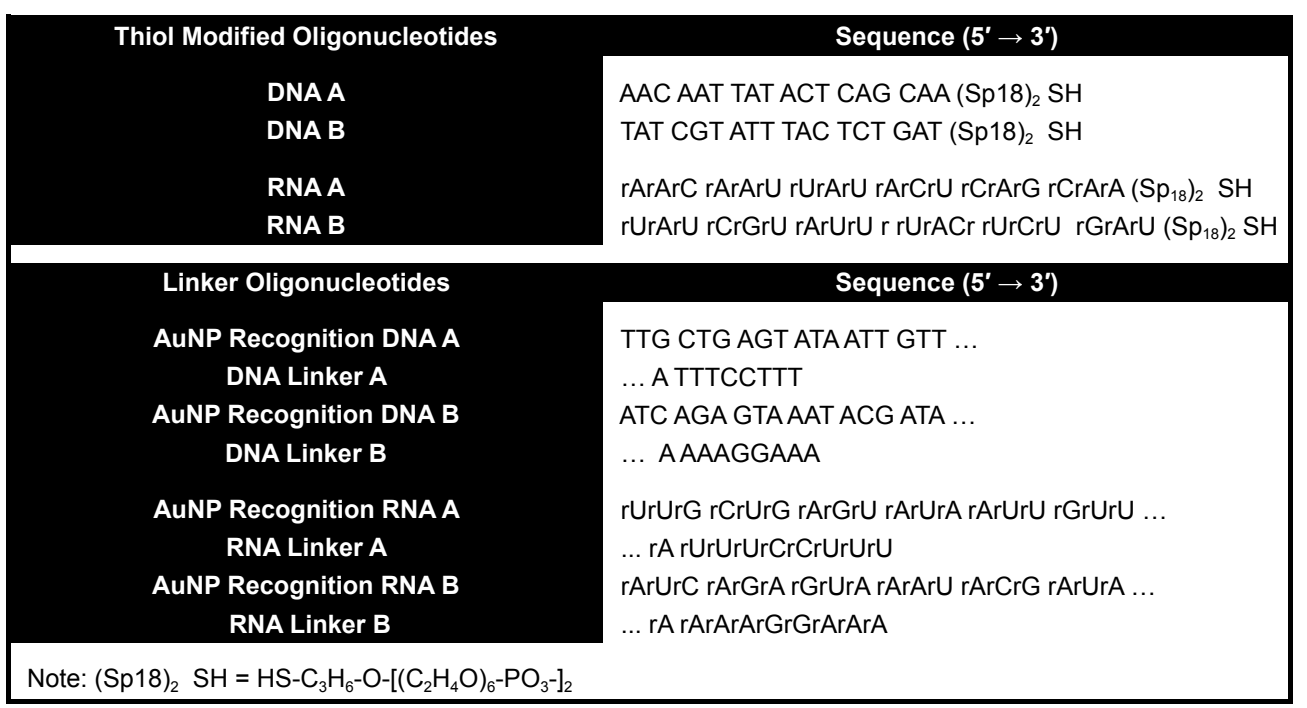

Table S1. List of sequences used to assemble nanoparticle superlattices.

\begin{tabular}{|c|c|c|}
\hline$\%$ Particle A & $\%$ Particle B & \% Particle B' \\
\hline 100 & 0 & 100 \\
\hline 100 & 1 & 99 \\
\hline 100 & 5 & 95 \\
\hline 100 & 10 & 90 \\
\hline 100 & 20 & 80 \\
\hline 100 & 30 & 70 \\
\hline 100 & 40 & 60 \\
\hline 100 & 50 & 50 \\
\hline 100 & 60 & 40 \\
\hline 100 & 70 & 30 \\
\hline 100 & 80 & 20 \\
\hline 100 & 90 & 10 \\
\hline 100 & 95 & 5 \\
\hline 100 & 99 & 1 \\
\hline 100 & 100 & 0 \\
\hline
\end{tabular}

Table S2. Design of nanoparticle superlattices with controlled ratios of particle B:B'. 


\section{Before Thermal-Annealing}

a)

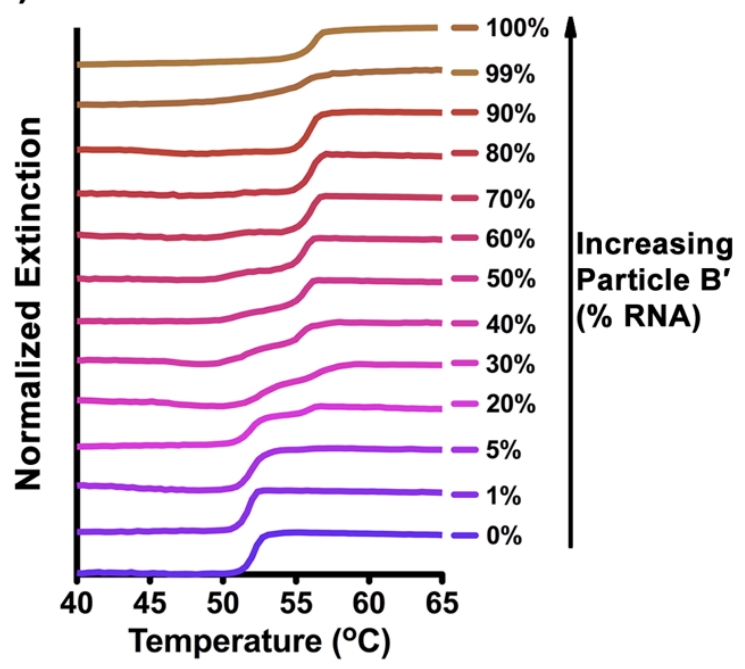

b)

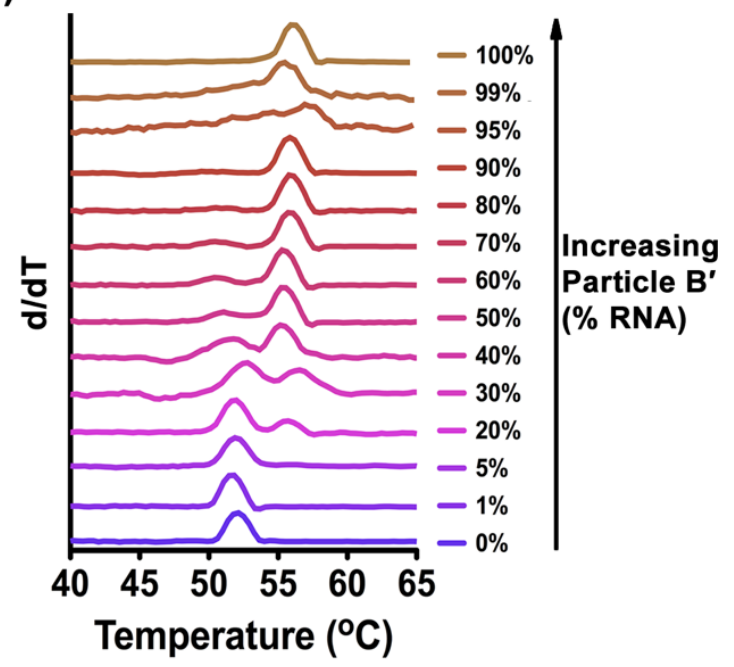

After Thermal-Annealing
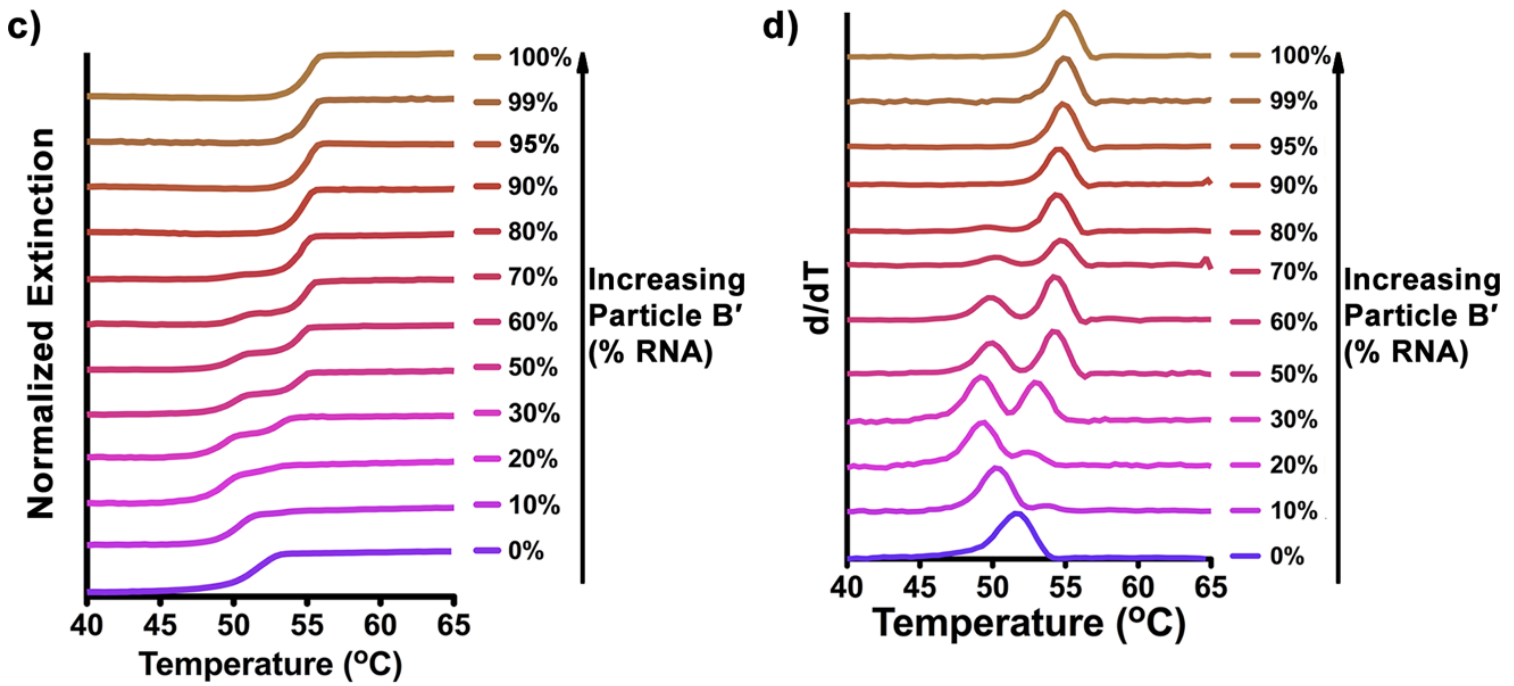

Figure S1. Melting profiles before (a) and after (c) thermal annealing. The first derivatives are plotted in (b) and (d). The samples post-thermal annealing should have fewer kinetic defects compared to the room temperature aggregated ones. It is worth noting that when taking the first derivative of the curves and observing two peaks in a plot of $\mathrm{d} / \mathrm{dT}$ versus temperature, the two observed melting transition temperatures are $\approx 1$ $3{ }^{\circ} \mathrm{C}$ different from the pure DNA/DNA and DNA/RNA melting transitions. This is due to the fact that these melting transitions are an average of the population, and in the nanoparticle crystals, there are different populations of DNA/DNA and DNA/RNA bonding elements. 

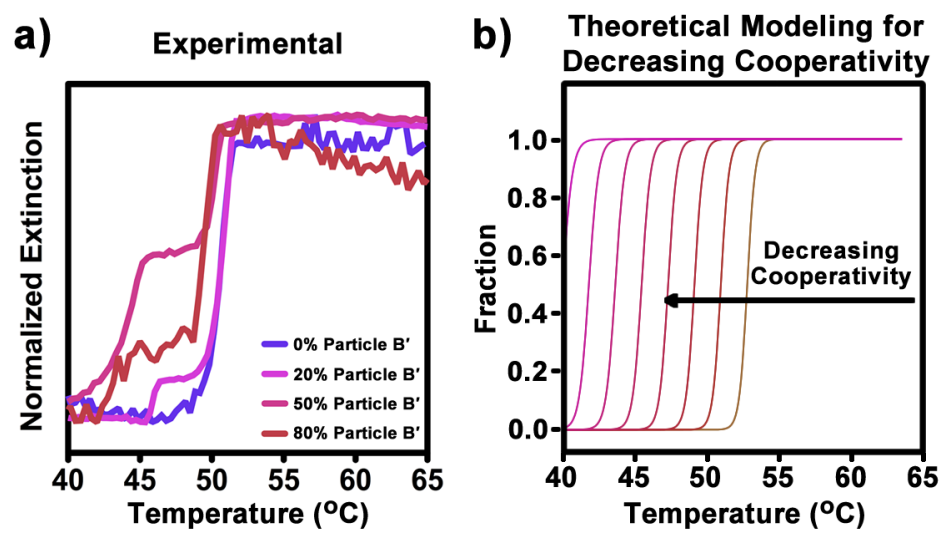

Figure S2. Theoretical and experimental melting transitions after enzymatic removal of particle $B^{\prime}$

a) Melting curves for nanoparticle superlattices after incubation with RNase A. The melting transition at $54{ }^{\circ} \mathrm{C}$ that was observed for the DNA/RNA bonding elements is no longer present. The melting transition observed at $\approx 52{ }^{\circ} \mathrm{C}$ corresponds to the DNA/DNA bonding elements. An additional melting transition shows up at $45{ }^{\circ} \mathrm{C}$. Theoretical modeling was used to confirm the origin of this transition (below).

b) Theoretical modeling of nanoparticle superlattices using the two-state melting model was utilized to explain the origin of the new melting transition at $45^{\circ} \mathrm{C}$ that was observed in (a). The extent of cooperativity (i) was decreased in 0.5 increments from 12.5 to 9 (right to left). Therefore, this suggests that the additional peak at $45^{\circ} \mathrm{C}$ seen in (a) results from DNA/DNA bonding elements in less cooperative environments. 


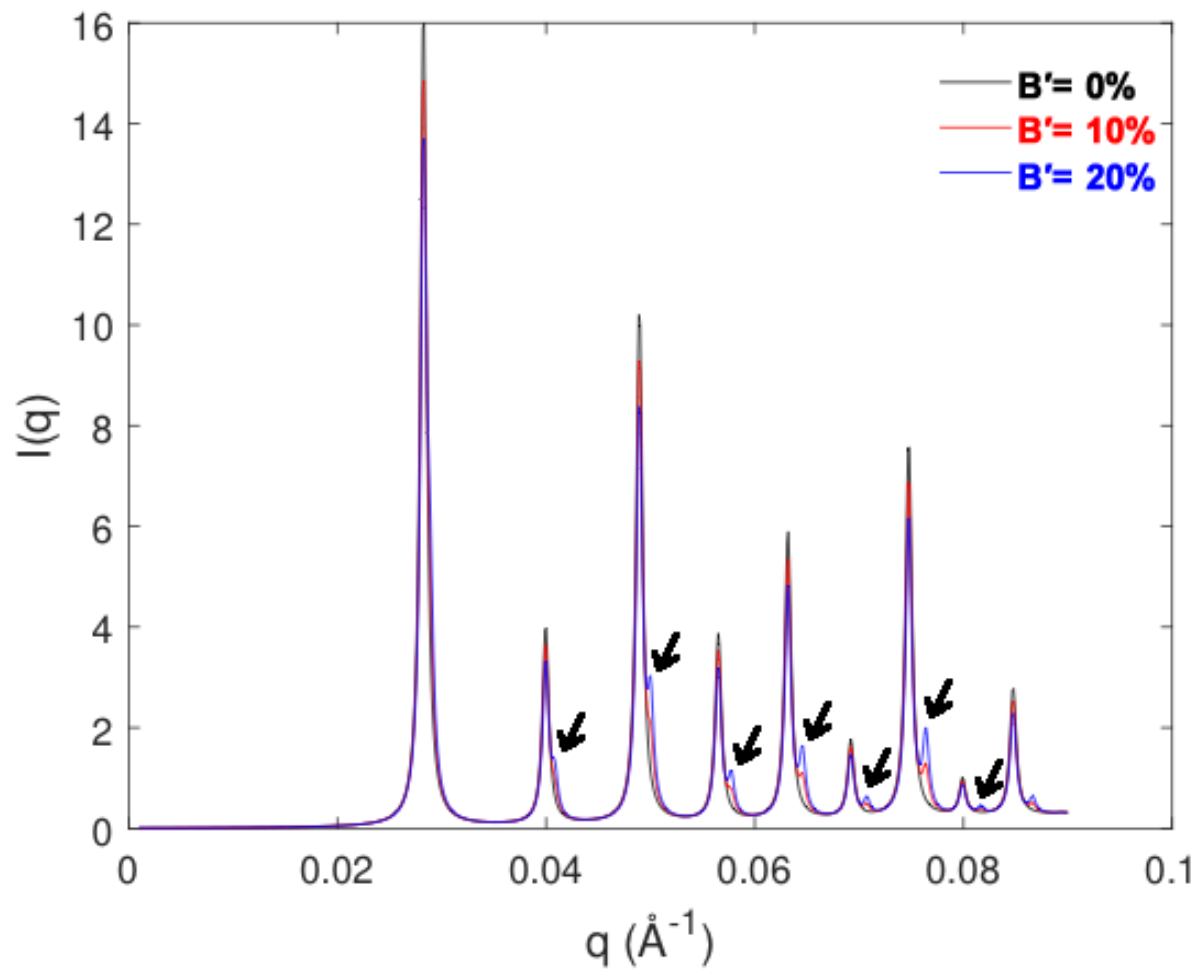

Figure S3. Theoretical SAXS scattering patterns for Structure 1

Structure 1 consists of phase separated crystalline populations of particles A, B and particles A, $\mathrm{B}^{\prime}$. In the above theoretically generated pattern, the percent of particle $\mathrm{B}^{\prime}$ is increased from 0 to $20 \%$. As indicated by the arrows, peak splitting and a second crystal domain is observed as there are two separate crystalline regions with slightly different lattice constants (see 0 and $100 \%$ data in Figure $3 a$ in the main text) when particle $\mathrm{B}^{\prime}$ is introduced. The peak splitting is more pronounced with higher percentages of particle B'. For the purposes of the model, the crystal domain size was set at $200 \mathrm{~nm}$. 

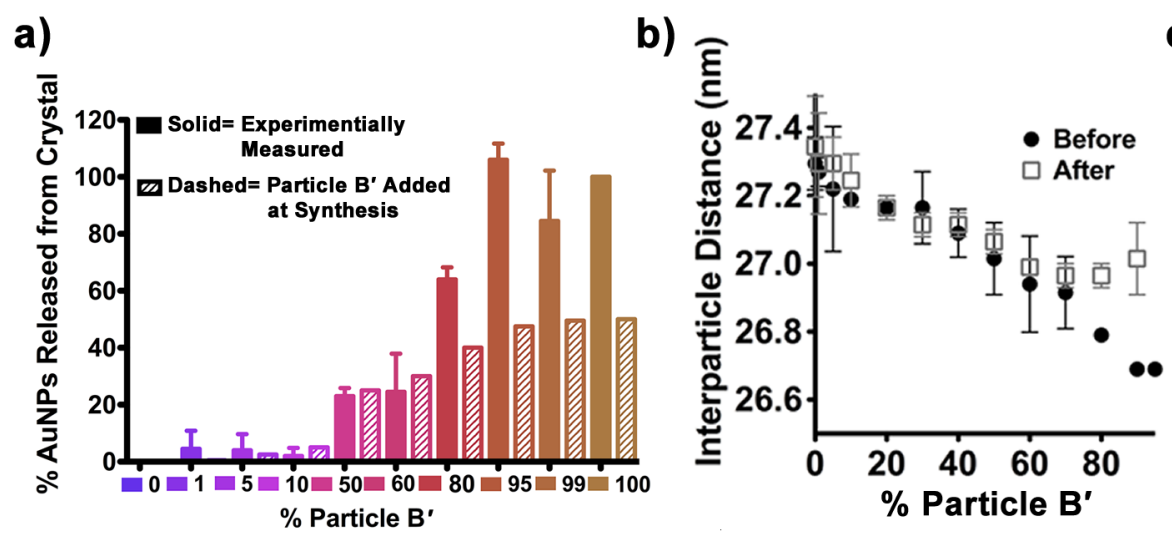

c)

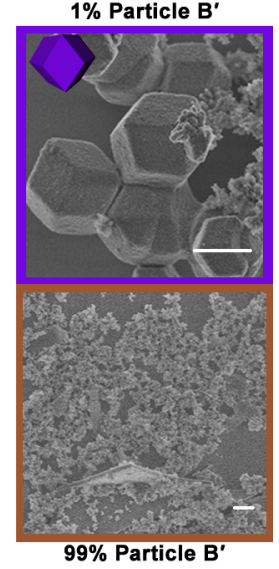

Figure S4. Change in interparticle distance after enzymatic removal of particle $B^{\prime}$

a) Measure of the supernatants in Figure $4 \mathrm{~b}$ using UV-visible spectroscopy. Error bars represent $n=2$ measurements. A description of the calculation is available in the Materials \& Methods.

b) Analysis of the data in Figure 5a yields a consistent decrease in interparticle distance with increasing percentage of particle $\mathrm{B}^{\prime}$. Up to $70 \%$ particle $\mathrm{B}^{\prime}$, there is little change in interparticle distance for the superlattices before and after interaction with RNase A.

c) SEM micrographs of nanoparticle superlattices after RNase A incubation. Single crystal rhombic dodecahedra persist at low particle $\mathrm{B}^{\prime}$ ratios $(<10 \%)$, followed by loss of superstructure to yield particles free in solution at high ratios of particle $\mathrm{B}^{\prime}$ $(>80 \%)$. Scale bars $=1 \mu \mathrm{m}$. 

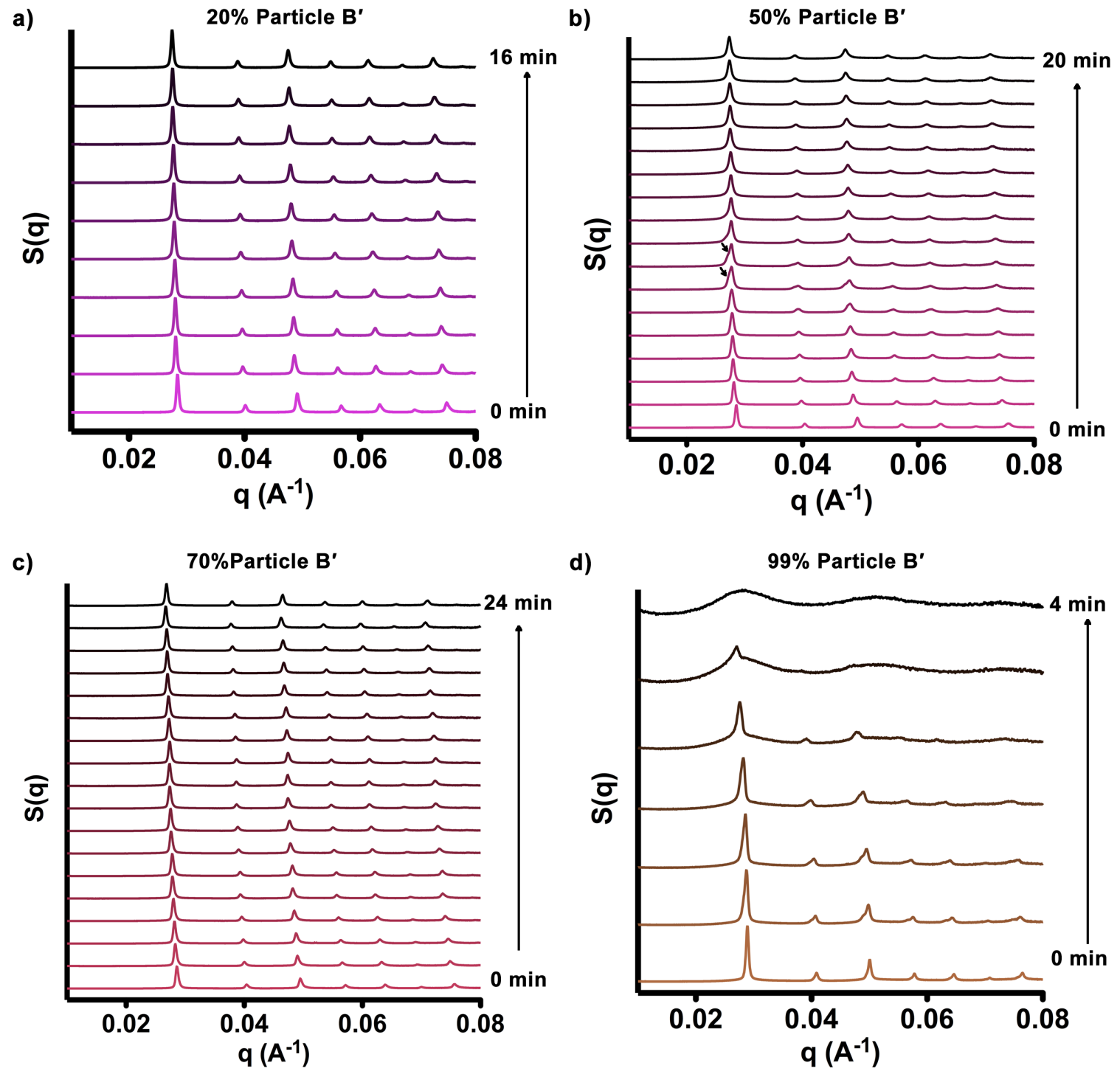

Figure S5. In situ small-angle X-ray scattering (SAXS) for the interaction of RNase A $(4 \boldsymbol{\mu g})$ with nanoparticle superlattices at various ratios of DNA:RNA particle B
a) $20 \%$ particle $\mathrm{B}^{\prime}$
b) $50 \%$ particle $\mathrm{B}^{\prime}$. Slight peak splitting is observed transiently in the case of $50 \%$ particle B' (indicated by the arrows), suggesting that at the highest ratio of particle $\mathrm{B}: \mathrm{B}^{\prime}$ the crystal may be fractioning into two upon addition of the enzyme.
c) $70 \%$ particle $\mathrm{B}^{\prime}$
d) $99 \%$ particle $\mathrm{B}^{\prime}$ 

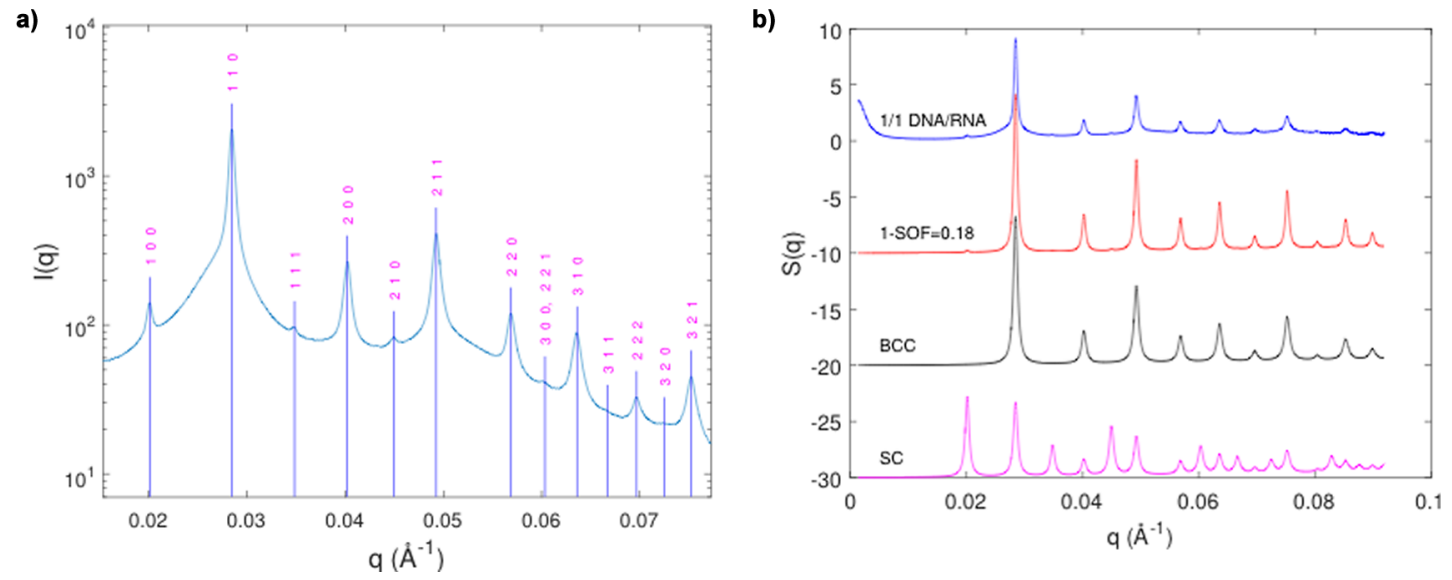

Figure S6. Peak fitting of nanoparticle superlattices

a) Representative SAXS pattern for nanoparticle superlattices with 1:1 particle B:B' fit to bcc and simple cubic crystal lattice symmetry.

b) (Bottom to top): Simulated scattering pattern for a simple cubic lattice; Simulated scattering pattern for a bcc lattice; Simulated scattering pattern for $1-\mathrm{SOF}=0.18$; Experimental scattering pattern for nanoparticle superlattices with 1:1 ratio of particle $B: B^{\prime}$ 


\section{SI Materials and Methods}

Preparation of Sterile, RNase-Free Solutions

To prepare sterile solutions of water, 1x phosphate buffered saline (PBS; Corning), and $5 \mathrm{M}$ sodium chloride ( $\mathrm{NaCl}$; Sigma-Aldrich), $1 \mathrm{~mL}$ of diethylprocarbonate (DEPC; Sigma-Aldrich) was added to $1 \mathrm{~L}$ of the aforementioned solution. The mixture was then shaken at $50 \mathrm{rpm}$ for 2 hours at $45^{\circ} \mathrm{C}$ before being autoclaved.

\section{Synthesis of DNA and RNA}

DNA oligonucleotides were synthesized on an MM48 DNA synthesizer (BioAutomation) with reagents obtained from Glen Research or ChemGenes. RNA oligonucleotides were synthesized using 2'-O [(triisopropylsilyl)oxy] methyl-RNA phosphoramidites (ChemGenes) on a MerMade 12 system (Bioautomation) according to the manufacturer recommended cleavage and deprotection protocols. All oligonucleotides were purified using reverse-phase high-performance liquid chromatography (RP-HPLC) on a Varian Microsorb C18 column $(10 \mu \mathrm{M} ; 300 \AA \AA \sim 10$ $\mathrm{mm}$ ) with $0.1 \mathrm{M}$ triethylammonium acetate (TEAA) at $\mathrm{pH} 7$ with a $1 \%$ gradient of $100 \%$ $\mathrm{CH}_{3} \mathrm{CN}$ at a flow rate of $3 \mathrm{~mL} / \mathrm{min}$, while monitoring the UV signal of the nucleic acids at $254 \mathrm{~nm}$. After purification, the oligonucleotides were lyophilized and stored at $-80^{\circ} \mathrm{C}$.

\section{Functionalization of Nanoparticles with DNA or RNA}

$10 \mathrm{~nm}$ gold nanoparticles (AuNPs) were purchased from Ted Pella and used as received. DNA and RNA functionalization was conducted according to literature procedures for functionalization of DNA and RNA. ${ }^{3-5}$ Briefly, oligonucleotides synthesized with a propyl-thiol modified 3' terminus were treated with $100 \mathrm{mM}$ dithiothreitol (DTT; Sigma-Aldrich) for one hour at room temperature to reduce the disulfide, at which point the DTT was removed via size-exclusion chromatography with a NAP5 or NAP10 column (GE Healthcare). The concentration of the oligonucleotides was confirmed using a Cary5000 UV-Vis spectrometer (Cary) and then added to gold nanoparticle suspensions at a ratio of 1 optical density (OD) unit of DNA or RNA per 1 $\mathrm{mL}$ of $10 \mathrm{~nm}$ AuNPs (approximately $3 \mathrm{nmol}$ of DNA or RNA per $1 \mathrm{~mL}$ of AuNPs) and placed on a rotary shaker (Sigma Aldrich). After incubation for 12 hours, the solution was brought to $100 \mathrm{mM}$ phosphate buffer $(\mathrm{pH}=7.4)$ and left on a rotary shaker for 1 hour, at which point the solution was brought to $0.1 \%$ sodium dodedcyl sulfate (SDS; Ambion) and left on the rotary shaker for an additional $30 \mathrm{~min}$. The solution was slowly salted from $0-0.5 \mathrm{M} \mathrm{NaCl}$ using a stock solution of $5 \mathrm{M} \mathrm{NaCl}$, where the salt was raised $0.05-0.1 \mathrm{M}$ every $30 \mathrm{~min}$. Once the solution was at $0.5 \mathrm{M} \mathrm{NaCl}$, it was placed on the rotary shaker for 12 hours. Excess DNA or RNA was removed via centrifugation using Amicon Ultra-15 centrifugal filter unit (50-kDa cutoff; EMD Millipore) for $10 \mathrm{~min}$ in a swinging bucket rotor at $1,500 \mathrm{x}$. The flow-through was removed and the particles were washed four times with $10 \mathrm{mM}$ phosphate buffer, $0.5 \mathrm{M} \mathrm{NaCl}$, and $0.1 \%$ SDS in water and centrifuged in an Eppendorf tube for $90 \mathrm{~min}$ at $21,000 \mathrm{x} \mathrm{g}$. The supernatant was removed and the pellet was re-suspended in the same solution. The AuNP concentration was measured by absorbance at $520 \mathrm{~nm}\left(\mathrm{E}_{520 \mathrm{~nm}}=9.55 \times 10^{8} \mathrm{M}-1 \mathrm{~cm}-1\right)$ using a Cary $5000 \mathrm{UV}$-vis. The SNAs were stored at $4{ }^{\circ} \mathrm{C}$ when not in use. All solutions used were DEPC-treated prior to use. 


\section{Addition of Linker Strands to AuNPs with DNA and RNA}

Hybridization of linker strands that present short sticky ends at the periphery of the particles' hydrodynamic radii of DNA and RNA must occur onto the thiololigonucleotide functionalized AuNPs. ${ }^{3-5}$ The linkers (Table S1) were allowed to hybridize for $\geq 1$ hour while shaking at $37^{\circ} \mathrm{C}$ in a thermomixer to facilitate the formation of double stranded DNA and RNA duplexes consisting of the oligonucleotide linker and its complementary sequence (duplex). The duplexed linkers (as well as the non-duplexed short linkers) were added to the AuNPs at 120 equivalents (relative to the number of moles of $\mathrm{Au}$ ). Particles $\mathrm{A}, \mathrm{B}$, and $\mathrm{B}^{\prime}$ with respective linkers were prepared at $50 \mathrm{nM}$ final AuNP concentration.

\section{Aggregation of Particles $A, B$ and Particles $A, B^{\prime}$}

Particles $B$ and $\mathrm{B}^{\prime}$ were mixed in the appropriate ratio (Table S2) and then vortexed to ensure full mixing. An equal volume of particle A (to that of the volume of particle $\mathrm{B}+\mathrm{B}^{\prime}$ ), was then added, and the mixture was sonicated and then allowed to sit at room temperature for 1-2 hours to allow the particles to aggregate and precipitate out of solution.

\section{Melting Experiments}

Melting curves were measured with a Cary 5000 UV-Vis-NIR spectrophotometer (Agilent; Santa Clara, CA) equipped with a multi-cell holder with thermal control. Each sample was prepared by transferring $30 \mu \mathrm{L}$ of dispersed aggregates or crystals $(50 \mathrm{nM}$ AuNP particle A $+50 \mathrm{nM}$ particle B, B') to $900 \mu \mathrm{L}$ of $0.5 \mathrm{M} \mathrm{NaCl}, 10 \mathrm{mM}$ phosphate buffer, $0.1 \%$ SDS in water in an absorbance cuvette (Hellma Cells Inc.). While stirring, the temperature was increased from $25{ }^{\circ} \mathrm{C}$ to $70{ }^{\circ} \mathrm{C}$ at a rate of $0.2{ }^{\circ} \mathrm{C} / \mathrm{min}$ while monitoring the extinction of the solution at 260 and $520 \mathrm{~nm}$ with the same data collection interval. As the temperature increased, the aggregates broke apart due to the dehybridization of the oligonucleotide duplexes formed between the sticky ends of adjacent, complementary particles. The resulting free particles no longer have dampened optical signals as when aggregated, which leads to an increase in the extinction at 260 and $520 \mathrm{~nm}$, allowing for a determination of the melting temperature by taking the first derivative of the melting curve and finding the maximum value.

\section{Two State Thermodynamic Melting Model}

Such a two-state melting model can be represented as:

$$
f=\frac{1}{1+\exp \left[\frac{\Delta H_{\text {total }}}{R}\left(\frac{1}{T}-\frac{1}{T_{\mathrm{m}}}\right)\right]}
$$

where

$$
\Delta H_{\text {total }}=\sum_{i} \Delta H_{i}
$$

and 


$$
T_{\mathrm{m}}=\frac{\Delta H_{\text {total }}}{\sum_{i} \frac{\Delta H_{i}}{T_{\mathrm{m}, i}}}
$$

where $\mathrm{f}$ is the fraction melted, $\Delta \mathrm{H}_{\text {total }}$ is the total enthalpy of melting, $\Delta \mathrm{H}_{i}$ is the enthalpy of a single oligonucleotide melting transition, $\mathrm{R}$ is the gas constant, and $\mathrm{T}_{\mathrm{m}}$ is the melting transition temperature. From this model, it can be inferred that $\Delta \mathrm{H}_{\text {total }}$ is responsible for the melting transition width while $T_{m}$ is the weighted average of the individual strand melting temperatures.

The $\mathrm{T}_{\mathrm{m}, i}$ and $\Delta \mathrm{H}_{i}$, the melting temperature and enthalpy of melting for a single DNA sticky end connection (TTTCCTTT) respectively, were calculated using the nearest neighbor model. ${ }^{6,7}$ For each system, $T_{\mathrm{m}}$ takes the general form ${ }^{T}=T_{\mathrm{m}, i}+(i-1) \alpha$, where $\mathrm{T}_{\mathrm{m}}$ is the collective melting temperature, $\mathrm{T}_{\mathrm{m}, i}$ is the melting temperature of an individual sticky end, $i$ is the number of connections, and $\alpha$ is a scaling factor. The number of oligonucleotide connections $(i)$ is set as the maximum number of possible connections by a particle (100 strands per nanoparticle) divided by the number of nearest neighbors in a bcc crystal (8). For the DNA system, $\mathrm{T}_{\mathrm{m}, i}$ is $6.8^{\circ} \mathrm{C}, \Delta \mathrm{H}_{i}=60.5 \mathrm{kcal} / \mathrm{mol}$, and $\alpha$ is 3.8 . For the RNA system, $\mathrm{T}_{\mathrm{m}, i}$ is $8.7^{\circ} \mathrm{C}, \Delta \mathrm{H}_{i}=58.6 \mathrm{kcal} / \mathrm{mol}$, and $\alpha$ is 3.9 . These values gave good agreement with the experimental cooperative values (i.e. melting temperatures) of 50.5 and $53.0{ }^{\circ} \mathrm{C}$ for the DNA/DNA and DNA/RNA systems, respectively. For the homogeneously mixed DNA/DNA, DNA/RNA systems, $\mathrm{T}_{\mathrm{m}}$ was calculated as fractional variation between the pure DNA and RNA cases

$T_{\mathrm{m}}=\mathrm{X}[6.8+(i-1) 3.8]+(1-\mathrm{X})[8.7+(i-1) 3.9]$

where $\mathrm{X}$ is the fraction of DNA. In these structures, $\mathrm{T}_{\mathrm{m}}$ is varied linearly as a function of DNA/RNA composition. For the phase separated systems, the melting behavior is assumed to be a linear combination of the distinct DNA/DNA and DNA/RNA models, where

$T_{\mathrm{m}}=\mathrm{X}[\operatorname{frac} D N A-D N A]+(1-\mathrm{X})[\operatorname{frac} D N A-R N A]$

where frac DNA-DNA and frac DNA-RNA were calculated using the parameters described above. Here $T_{m}$ is a linear combination of the fraction of the aggregate remaining after application of the model; because the DNA and RNA rich systems exhibit distinct $T_{m}$ values, two melting transitions are seen in the collective behavior.

\section{Slow-Cool Annealing of Nanoparticle Superlattices}

$100 \mu \mathrm{L}$ of dispersed superlattices were transferred to $200 \mu \mathrm{L}$ polymerase chain reaction (PCR) strip tubes (Eppendorf). The samples were brought to a temperature 5-10 ${ }^{\circ} \mathrm{C}$ above their melting temperature $\left(60^{\circ} \mathrm{C}\right)$ and then slowly cooled to $25^{\circ} \mathrm{C}$ at a rate of $0.01{ }^{\circ} \mathrm{C} / \mathrm{min}$ using a ProFlex ${ }^{\mathrm{TM}}$ PCR system (Applied Biosystems). The slow cooling took 3 days to complete. 
Small-Angle X-Ray Scattering (SAXS)

All synchrotron SAXS experiments were conducted at the Dow-NorthwesternDupont Collaborative Access Team (DND-CAT) Beamline 5ID-D at the Advanced Photon Source (APS) at Argonne National Laboratory. Experiments were collected with $10 \mathrm{keV}$ (wavelength $\lambda=1.24 \AA$ ) collimated X-rays calibrated against a silver behenate standard. Exposure times of 0.1 and 1 second were used. Approximately $30 \mu \mathrm{L}$ of slowcooled nanoparticle superlattices were loaded into $1.5 \mathrm{~mm}$ quartz capillary tubes (Charles Supper) and placed into a sample stage in the path of the X-ray beam. Two-dimensional scattering data were collected on a CCD area detector and converted to 1D data by taking a radial average of the $2 \mathrm{D}$ data to generate plots of scattering intensity $\mathrm{I}(\mathrm{q})$ as a function of the scattering vector $\mathrm{q}(\mathrm{q}=4 \pi \sin \theta / \lambda$, where $\theta$ and $\lambda$ are the scattering angle and wavelength of the X-rays used, respectively). The particle form factor, $\mathrm{P}(\mathrm{q})$, the scattering due to individual dispersed particles in solution, was subtracted from the experimental data to obtain the lattice structure factor, S(q). Form factor subtraction, radial averaging, and other SAXS data analysis were performed in Igor using the Irena and Nika macros (available free of charge from the APS at usaxs.xray.aps.anl.gov/staff/ilavsky/irena.html).

\section{Calculation of Interparticle Distance from the SAXS data}

The nearest neighbor distance, $d$ (in $\mathrm{nm}$ ), between particles in a superlattice can be calculated from the position of the first scattering peak, $\mathrm{q}_{0}$, in the scattering pattern using the following relationship for bcc crystallographic symmetry:

$$
d=\frac{0.1 \cdot \sqrt{6} \cdot \pi}{q_{0}}
$$

Calculation of the Domain Size from the SAXS Data

The crystal domain size $(t)$ was calculated using the Scherrer formula:

$$
t=\frac{0.9 \lambda}{\beta \cos \theta}
$$

where $\lambda=$ the wavelength of scattered X-rays, $\theta=$ the diffraction angle associated with the $q_{o}$ peak, and $\beta=$ the angular full width at half maximum of the $\mathrm{q}_{0}$ peak.

\section{Interaction of Nanoparticle Superlattices with RNase A ("Static" Experiment)}

To $30 \mu \mathrm{L}$ of nanoparticle superlattices, $1 \mu \mathrm{L}$ of RNase A ([RNase] $=1 \mu \mathrm{g} / \mu \mathrm{L}$ ) was added and subsequently vortexed for 15 seconds then incubated at room temperature for 12 hours. For analysis, the supernatant was removed and the concentration was measured using UV-visible spectroscopy to generate the data in Figure S3a. Each solid bar was generated by dividing by the concentration of AuNPs at $100 \%$ particle $\mathrm{B}^{\prime}$ to generate the "\% AuNPs in supernatant". The dashed bars are a calculation for the experimental concentration of particle $\mathrm{B}^{\prime}$ added at synthesis. For example, for $50 \%$ particle $\mathrm{B}^{\prime}$, this represents $25 \%$ of the AuNPs in the superlattice. After removal of the supernatant, the pellet was then re-suspended in $30 \mu \mathrm{L}$ of $10 \mathrm{mM}$ phosphate buffer, $0.1 \%$ 
SDS, and $0.5 \mathrm{M} \mathrm{NaCl}$ and run in a melting experiment, similar to what was described above to generate the data in Figure S2a. For the SAXS experiments in Figure 5a, $30 \mu \mathrm{L}$ of nanoparticle superlattices with $1 \mu \mathrm{L}$ of RNase A were loaded into capillaries and analyzed as is.

\section{Determination of the Structure Occupancy Factor (SOF)}

Scattering amplitude of a diffraction peak $h k l$ is described as:

$$
F_{h k l}=\sum_{j=1}^{m} N_{j} F_{j}\left(q_{h k l}\right) e^{2 \pi i\left(h x_{j}+k y_{j}+l z_{j}\right)},
$$

where $N_{j}$ is the site occupancy factor of a particle $j$ located at a fractional coordinate $\left(x_{j}\right.$, $\left.y_{j}, z_{j}\right) . F_{j}\left(q_{h k l}\right)$ is the form factor of the particle at $q=q_{h k l}$. The full scattering intensity curve or structure factor is then calculated using other equations described in Senesi et $a l{ }^{8}$ In this work, all compositions possess cubic unit cells except for disordered samples. Particle $\mathrm{A}$ and $\mathrm{B}, \mathrm{B}^{\prime}$ will be located at $(0,0,0)$ or $(1 / 2,1 / 2,1 / 2)$ positions, respectively. The SOF for particle $A$ is always 1 while that for particle $B, B^{\prime}$ may vary with the composition of DNA/RNA (i.e. ratio of particle $\mathrm{B}, \mathrm{B}^{\prime}$ ). We first simulated structure factors by varying the SOF for B and obtained the intensity ratios of (100) and (110) reflections as a function of the SOF for the $\mathrm{B}, \mathrm{B}^{\prime}$ site. Then, we curve-fitted the integrated intensities of the two peaks from experimental structure factors and calculated the experimental intensity ratios. By comparing the experimental ratios to the simulated ratios, the $\mathrm{SOF}$ values for the particle $\mathrm{B}, \mathrm{B}^{\prime}$ sites were obtained.

\section{In-Situ Flow Cell SAXS}

Time-dependent SAXS experiments were conducted using a flow cell set-up at 20 ${ }^{\circ} \mathrm{C}$ by mondification of previous method. ${ }^{5}$ To obtain a baseline reading, SAXS patterns were first collected for $80 \mu \mathrm{L}$ of nanoparticle superlattices. After the collection, the superlattices were then dispensed into an Eppendorf tube, which contained $10 \mu \mathrm{L}$ of ribonuclease (RNase) A at desired concentrations. At this point, a $5 \mu \mathrm{L}$ air bubble was pulled into the system, followed by $80 \mu \mathrm{L}$ of sample, and then another $5 \mu \mathrm{L}$ air bubble. Once the hutch was closed, oscillation of the sample between the two air bubbles at 10 $\mu \mathrm{L} /$ second began in order to allow the sample to mix. The sample was then shot with the X-ray beam approximately every 30 seconds. For all time-resolved measurements, the exposure time was 0.5 seconds. All tubing was rinsed with Nanopure water in between each run.

\section{Silica Embedding}

Following slow-cool annealing, the solution phase lattices were transferred to the solid state via silica encapsulation using a modified procedure from that described in Auyeung et al. ${ }^{9,10} 50 \mu \mathrm{L}$ of the slow-cooled samples (eg. lattices containing approximately $50 \mathrm{nM}$ of AuNPs) were transferred to a $1.5 \mathrm{~mL}$ Eppendorf tube and then the volume was raised to $500 \mu \mathrm{L}$ with a solution of $0.5 \mathrm{M} \mathrm{NaCl} .1 \mu \mathrm{L}$ of the quaternary ammonium salt, N-trimethoxysilylpropyl-N,N,N-trimethylammonium chloride (TMSPA), was added to the solution of nanoparticle assemblies and the tube was placed 
on a thermomixer, shaking at $300 \mathrm{rpm}$ at $20^{\circ} \mathrm{C}$. The tube was left shaking for $20 \mathrm{~min}$ to allow the TMSPA to electrostatically associate to the negatively-charged phosphate backbone of the DNA and RNA. Subsequently, $2 \mu \mathrm{L}$ of triethoxysilane (TES) was added to the solution to initiate silica growth first around the DNA and RNA where the TMPSA has associated and eventually around the entire lattices. Both the TMSPA and the TES were added in a large excess relative to the calculated number of phosphate groups on the DNA and RNA contained in the superlattice samples. The mixture was left on the thermomixer at $20^{\circ} \mathrm{C}$ for 24 hours, during which a cloudy precipitate formed above the nanoparticle aggregate that is presumably silica that has undergone bulk precipitation in solution. After 24 hours, the samples were briefly sonicated, then centrifuged at 15000 rpm for 5 seconds, at which point the solution was then removed and filled with $1 \mathrm{~mL}$ of DEPC-treated Nanopure water. This process was repeated four times, and after the final removal of the supernatant, the superlattices were re-suspended in $50 \mu \mathrm{L}$ of Nanopure water.

\section{Scanning Electron Microscopy (SEM)}

SEM images were obtained at the Northwestern University Atomic and Nanoscale Characterization Experimental Center (NUANCE) on a Hitachi SEM SU8030 instrument at an accelerating voltage of $3 \mathrm{kV} .5 \mu \mathrm{L}$ of solid-state silica embedded lattices in water were directly drop cast onto a silicon wafer (Nova Electronics) that was cut with a diamond scribe (Ted Pella) to a size appropriate for imaging.

\section{References}

(1) Jin, R.; Wu, G.; Li, Z.; Mirkin, C. A.; Schatz, G. C. J. Am. Chem. Soc. 2003, 125, 1643.

(2) Larkins, F. P.; Stoneham, A. M. J. Phys. C. 1971, 4, 143.

(3) Macfarlane, R. J.; Jones, M. R.; Lee, B.; Auyeung, E.; Mirkin, C. A. Science 2013, 341, 1222.

(4) Hurst, S. J.; Lytton-Jean, A. K.; Mirkin, C. A. Anal. Chem. 2006, 78, 8313.

(5) Barnaby, S. N.; Thaner, R. V.; Ross, M. B.; Brown, K. A.; Schatz, G. C.; Mirkin, C. A. J. Am. Chem. Soc. 2015, 137, 13566.

(6) Sugimoto, N.; Nakano, S.-i.; Katoh, M.; Matsumura, A.; Nakamuta, H.; Ohmichi, T.; Yoneyama, M.; Sasaki, M. Biochemistry 1995, 34, 11211.

(7) Sugimoto, N.; Nakano, S.; Yoneyama, M.; Honda, K. Nucleic Acids Res. 1996, 24, 4501 .

(8) Senesi, A. J.; Lee, B. J. Appl. Crystallogr. 2015, 48, 1172.

(9) Auyeung, E.; Li, T. I. N. G.; Senesi, A. J.; Schmucker, A. L.; Pals, B. C.; de la Cruz, M. O.; Mirkin, C. A. Nature 2014, 505, 73.

(10) Auyeung, E.; Macfarlane, R. J.; Choi, C. H.; Cutler, J. I.; Mirkin, C. A. Adv. Mater. 2012, 24, 5181. 